\title{
Radical Prostatectomy or Watchful Waiting in Early Prostate Cancer
}

Anna Bill-Axelson, M.D., Ph.D., Lars Holmberg, M.D., Ph.D., Hans Garmo, Ph.D., Jennifer R. Rider, Sc.D., Kimmo Taari, M.D., Ph.D., Christer Busch, M.D., Ph.D., Stig Nordling, M.D., Ph.D., Michael Häggman, M.D., Ph.D., Swen-Olof Andersson, M.D., Ph.D., Anders Spångberg, M.D., Ph.D., Ove Andrén, M.D., Ph.D., Juni Palmgren, Ph.D., Gunnar Steineck, M.D., Ph.D., Hans-Olov Adami, M.D., Ph.D., and Jan-Erik Johansson, M.D., Ph.D. Departments of Surgical Sciences (A.B.-A., M.H.) and Immunology, Genetics, and Pathology (C.B.), and the Regional Cancer Center Uppsala Örebro (L.H., H.G.), Uppsala University Hospital, Uppsala, the School of Health and Medical Sciences, Örebro University and Department of Urology, Örebro University Hospital, Örebro (S.-O.A., O.A., J.-E.J.), the Department of Urology, Linköping University Hospital, Linköping (A.S.), the Department of Oncology and Pathology, Division of Clinical Cancer Epidemiology (G.S.), and Department of Medical Epidemiology and Biostatistics (J.P., H.-O.A.), Karolinska Institutet, Stockholm, and the Division of Clinical Cancer Epidemiology, Sahlgrenska Academy, Gothenburg (G.S.) - all in Sweden; King's College London, School of Medicine, Division of Cancer Studies, London (L.H., H.G.); Channing Laboratory, Department of Medicine, Brigham and Women's Hospital and Harvard Medical School (J.R.R.), and the Department of Epidemiology, Harvard School of Public Health (J.R.R., H.-O.A.) - all in Boston; and the Department of Urology, Helsinki University Central Hospital (K.T.), and the Department of Pathology, University of Helsinki (S.N.) - both in Helsinki

\section{Abstract}

BACKGROUND—Radical prostatectomy reduces mortality among men with localized prostate cancer; however, important questions regarding long-term benefit remain.

METHODS-Between 1989 and 1999, we randomly assigned 695 men with early prostate cancer to watchful waiting or radical prostatectomy and followed them through the end of 2012. The primary end points in the Scandinavian Prostate Cancer Group Study Number 4 (SPCG-4) were death from any cause, death from prostate cancer, and the risk of metastases. Secondary end points included the initiation of androgen-deprivation therapy.

RESULTS-During 23.2 years of follow-up, 200 of 347 men in the surgery group and 247 of the 348 men in the watchful-waiting group died. Of the deaths, 63 in the surgery group and 99 in the watchful-waiting group were due to prostate cancer; the relative risk was 0.56 (95\% confidence interval $[\mathrm{CI}], 0.41$ to $0.77 ; \mathrm{P}=0.001$ ), and the absolute difference was 11.0 percentage points

Copyright (@) 2014 Massachusetts Medical Society.

Address reprint requests to Dr. Bill-Axelson at the Department of Urology, Uppsala University Hospital, 75185 Uppsala, Sweden, or at anna.bill.axelson@akademiska.se.

Drs. Bill-Axelson and Holmberg contributed equally to this article.

No potential conflict of interest relevant to this article was reported.

Disclosure forms provided by the authors are available with the authors with the full text of this article at NEJM.org. 
( $95 \%$ CI, 4.5 to 17.5 ). The number needed to treat to prevent one death was 8 . One man died after surgery in the radical-prostatectomy group. Androgen-deprivation therapy was used in fewer patients who underwent prostatectomy (a difference of 25.0 percentage points; $95 \% \mathrm{CI}, 17.7$ to 32.3). The benefit of surgery with respect to death from prostate cancer was largest in men younger than 65 years of age (relative risk, 0.45 ) and in those with intermediate-risk prostate cancer (relative risk, 0.38). However, radical pros-tatectomy was associated with a reduced risk of metastases among older men (relative risk, $0.68 ; \mathrm{P}=0.04$ ).

CONCLUSIONS-Extended follow-up confirmed a substantial reduction in mortality after radical prostatectomy; the number needed to treat to prevent one death continued to decrease when the treatment was modified according to age at diagnosis and tumor risk. A large proportion of long-term survivors in the watchful-waiting group have not required any palliative treatment. (Funded by the Swedish Cancer Society and others.)

The scandinavian prostate cancer Group Study Number 4 (SPCG-4), a randomized trial of radical prostatectomy versus watchful waiting in men with localized prostate cancer diagnosed before the era of prostate-specific antigen (PSA) testing, showed a survival benefit of radical prostatectomy as compared with observation at 15 years of follow-up. ${ }^{1}$ By contrast, the Prostate Cancer Intervention versus Observation Trial (PIVOT), initiated in the early era of PSA testing, showed that radical prostatectomy did not significantly reduce prostate cancer-specific or overall mortality after 12 years. ${ }^{2}$ PSA screening profoundly changes the clinical domain of study. Among other considerations, the substantial additional lead time necessitates very long follow-up periods in the PIVOT trial to determine the effect of surgery as compared with observation. In the meantime, the SPCG-4 offers insight regarding the effectiveness of radical prostatectomy and the natural history of prostate cancer.

In an 18-year follow-up of SPCG-4, an accumulating number of events permitted analyses stratified according to age and tumor risk. Furthermore, we examined the prevalence of metastases and the use of palliative treatments to estimate the long-term disease burden according to study group.

\section{METHODS}

PATIENTS

Between October 1989 and December 1999, at 14 centers in Sweden, Finland, and Iceland, we randomly assigned 695 men with localized prostate cancer to either radical prostatectomy or watchful waiting, as described in previous articles, ${ }^{1,3,4}$ and in the study protocol (www.cancercentrum.se). The study was approved by the regional ethics committee for each participating center, and oral informed consent was obtained from all patients. All authors vouch for the accuracy and completeness of the data, and the last author vouches for the adherence of the study to the protocol.

Men were eligible for inclusion in the study if they were younger than 75 years of age and had a life expectancy of more than 10 years, had no other known cancers, and had a localized tumor of stage T0d (later named T1b), T1, or T2, as defined by the 1978 criteria of the International Union against Cancer. After the revision of staging criteria in 1987, T1c 
tumors were also included, starting in $1994 .^{5}$ Tumors had to be well differentiated to moderately well differentiated, according to the World Health Organization (WHO) classification, on the basis of core biopsies or fine-needle aspiration. All patients included in the study were required to have a serum PSA level of less than $50 \mathrm{ng}$ per milliliter and a negative bone scan.

\section{STUDY DESIGN}

In men assigned to the radical-prostatectomy group, the surgical procedure started with a lymphadenectomy of the obturator fossa. ${ }^{6}$ If no nodal metastases were detected in frozen sections, the radical prostatectomy was performed. ${ }^{7}$ Radical excision of the tumor was given priority over nerve-sparing surgery. Men who were assigned to observation did not receive any immediate treatment.

If signs of local recurrence (a palpable nodule or histologically confirmed recurrence) developed in a patient in the radical-prostatectomy group, androgen-deprivation therapy was initiated. Men in the watchful-waiting group who had signs of obstructive voiding were treated with transurethral resection. Confirmed metastases (detected by means of bone scans) were treated with hormone therapy. In 2003, clinicians were allowed to initiate androgen-deprivation treatment if there were signs of tumor progression or an increasing PSA level or if they believed that hormone therapy would provide a clinical benefit. In 1999, all core biopsy specimens were reviewed by four uropathologists and graded according to the Gleason system. ${ }^{8}$ (Before 2005, the Gleason score was the sum of the two most common histologic patterns or grades in a prostate tumor, each of which is graded on a scale of 1 to 5 , with 5 indicating the most aggressive pattern.)

Patients were followed every 6 months for 2 years and annually thereafter. At the last monitoring rounds, men who were unable to attend the clinic were followed from nursing homes or by their general practitioner with the use of PSA monitoring. Initially, bone scans were obtained annually, but after 2003, they were obtained every second year. Distant metastases were considered to be present when bone scans, skeletal radiographs, computed tomographic scans, or chest radiographs showed metastases or if lymph nodes at sites other than the regional sites showed cytologic or histologic evidence of prostate cancer. In the radical-prostatectomy group, local recurrence was defined as the presence of a palpable mass on digital rectal examination or a histologically confirmed tumor on rectal biopsy. In the watchful-waiting group, tumor progression was defined as palpable extracapsular extension or symptoms of obstructive voiding that required intervention.

In patients who died, an independent endpoint committee whose members were unaware of the study-group assignments determined the cause of death on the basis of information extracted from the patients' medical records. The committee members used a protocol that defined disease progression according to increases in PSA levels, local recurrence, the occurrence of metastases, and the need for androgen-deprivation therapy and palliative treatments. Each member of the end-point committee determined the cause of death; in cases in which there was disagreement among the members regarding the cause of death, the committee met to reach consensus. All participants were followed until December 31, 2012, and none of the patients were lost to follow-up. 


\section{STATISTICAL ANALYSIS}

Analyses have been performed every third year according to the protocol. The primary end points were death from any cause, death from prostate cancer (with death from other causes treated as a competing risk), and risk of metastases in bone, outside the pelvic area, or both. Secondary end points included initiation of androgen-deprivation therapy (with death from any cause treated as a competing risk).

We used Gray's test to assess treatment effects. ${ }^{9}$ Effect sizes were quantified both by analyzing relative risks with $95 \%$ confidence intervals and by determining differences in cumulative incidence (with 95\% confidence intervals). Relative risks were estimated with the use of Cox proportional-hazards models in cases in which proportionality was verified by means of visual inspection of the parallelisms of the logarithms of the estimated cumulative incidence. A cumulative incidence approach was used to account for competing risks among various causes of death. ${ }^{10}$

To assess the possible modification of the treatment effect, analyses were stratified according to the patient's age at diagnosis ( $<65$ years vs. $\ 65$ years) and tumor risk. The subgroup analyses were not included in the main protocol but were specified before the data were reviewed. Risk groups were defined with the use of Gleason scores from the pathological review as follows: low risk, PSA level less than 10 and either a Gleason score of less than 7 or WHO grade 1 (on a scale of 1 to 3, with higher grades indicating more aggressive disease) in tumors that were diagnosed only by means of cytologic assessment; high risk, PSA level of 20 or higher or a Gleason score greater than 7; and intermediate risk, all patients who did not fulfill the criteria for low or high risk. The modification of the effect of radical prostatectomy was tested in the Cox proportional-hazards model by including an interaction term between the subgroup category and randomization group.

The prevalence of the use of palliative treatment was calculated at every other year of follow-up, ending at 18 years after randomization. Palliative treatment was androgendeprivation treatment (antiandrogen therapy or gonadotropin-releasing hormone analogues or orchiectomy) in patients with or without verified metastases and in patients with metastases who had received other palliative treatment (external or internal palliative radiation therapy, laminectomy, or chemotherapy drugs).

\section{RESULTS}

A total of 347 men were randomly assigned to the radical-prostatectomy group, and 348 men were assigned to the watchful-waiting group. The baseline characteristics of the two groups were similar; the mean age of the men in both groups was 65 years. Only $12 \%$ of the patients had nonpalpable T1c tumors at the time of enrollment in the study. The mean PSA level was approximately $13 \mathrm{ng}$ per milliliter (Table S1 in the Supplementary Appendix, available with the full text of this article at NEJM.org). By December 31, 2012, a total of 294 men in the radical-prostatectomy group had undergone a radical prostatectomy, and 294 men in the watchful-waiting group had not received curative treatment. In the radicalprostatectomy group, 23 patients (16 patients in the high-risk group, 7 patients in the intermediate-risk group, and no patients in the low-risk group) had lymph node-positive 
disease and thus did not undergo radical prostatectomy (Fig. S1 in the Supplementary

Appendix). The median follow-up time was 13.4 years (range, 3 weeks to 23.2 years).

\section{MORTALITY}

A total of 447 of the 695 men enrolled in the study (64\%) had died by the end of 2012. This total included 200 men in the radical-prostatectomy group and 247 men in the watchfulwaiting group. The cumulative incidence of death at 18 years was $56.1 \%$ in the radicalprostatectomy group and $68.9 \%$ in the watchful-waiting group (a difference of 12.7 percentage points; $95 \%$ confidence interval $[\mathrm{CI}], 5.1$ to 20.3 ), corresponding to a relative risk of death in the radical-prostatectomy group of 0.71 (95\% CI, 0.59 to $0.86 ; \mathrm{P}<0.001$ ) (Fig. 1 and Tables 1 and 2). The number needed to treat to prevent one death at 18 years of follow-up was 8 . One man in the radical-prostatectomy group died after surgery.

By the end of 2012, a total of 63 men in the radical-prostatectomy group and 99 men in the watchful-waiting group had died from prostate cancer. The cumulative incidence of death from prostate cancer at 18 years was $17.7 \%$ in the radical-prostatectomy group and $28.7 \%$ in the watchful-waiting group (a difference of 11.0 percentage points; 95\% CI, 4.5 to 17.5), corresponding to a relative risk of death in the radical-prostatectomy group of 0.56 (95\% CI, 0.41 to $0.77 ; \mathrm{P}=0.001$ ) (Fig. 1 and Tables 1 and 2). The difference in mortality continued to increase from 9.6 deaths per 1000 person-years during 5 to 10 years of follow-up to 24.5 deaths per 1000 person-years in the 15-to-20-year follow-up interval.

In both older and younger men, death from other causes without evidence of metastases or the use of androgen-deprivation therapy was more common in the radical-prostatectomy group than in the watchful-waiting group (Fig. 1).

\section{DISTANT METASTASES}

Distant metastases were diagnosed in 89 men in the radical-prostatectomy group and in 138 men in the watchful-waiting group. The cumulative incidence of distant metastases at 18 years of follow-up was $26.1 \%$ in the radical-prostatectomy group and $38.3 \%$ in the watchfulwaiting group (a difference of 12.2 percentage points; $95 \%$ CI, 5.1 to 19.3), corresponding to a relative risk of distant metastases in the radical-prostatectomy group of 0.57 (95\% CI, 0.44 to $0.75 ; \mathrm{P}<0.001$ ) (Fig. 2 and Table 1 ).

\section{PALLIATIVE TREATMENT}

In all, 145 men in the radical-prostatectomy group and 235 in the watchful-waiting group received androgen-deprivation therapy. The cumulative incidence of the use of androgendeprivation therapy at 18 years was $42.5 \%$ in the radical-prostatectomy group and $67.4 \%$ in the watchful-waiting group (a difference of 25.0 percentage points; 95\% CI, 17.7 to 32.3), corresponding to a relative risk of the use of androgen-deprivation therapy in the radicalprostatectomy group of $0.49(95 \% \mathrm{CI}, 0.39$ to $0.60 ; \mathrm{P}<0.001)$. Other palliative treatments, such as radiation therapy, were less common in the radical-prostatectomy group than in the watchful-waiting group (49 vs. 63); 4 men in the radical-prostatectomy group underwent laminectomy because of metastases as compared with 9 in the watchful-waiting group. 
Chemotherapy was less frequently used in the radical-prostatectomy group (13 and 17 men, respectively) (Table 1, and Fig. S1 in the Supplementary Appendix).

The difference in the use of palliative treatment between the study groups increased with time. At 18 years of follow-up, approximately $60 \%$ of men in the watchful-waiting group, as compared with approximately $40 \%$ of men in the radical-prostatectomy group, had disease progression (with or without confirmed metastases) and received androgen-deprivation therapy and other palliative treatments. The pattern was the same in both age groups (Fig. $3)$.

\section{OUTCOMES ACCORDING TO AGE AND RISK CATEGORY}

Among men who were younger than 65 years of age, there was a significant absolute reduction in all three investigated end points: a reduction of 25.5 percentage points in overall mortality, 15.8 percentage points in death from prostate cancer, and 15.8 percentage points in the risk of metastases. In this age group, the number needed to treat to prevent one death from prostate cancer was 4 .

Among men 65 years of age or older at diagnosis, there was no significant reduction in mortality, but there was a significant absolute reduction of 8.9 percentage points in the risk of metastases. In this age group, more men in the watchful-waiting group than in the radicalprostatectomy group died from prostate cancer or from causes other than prostate cancer, but with metastases present (52 men in the watchful-waiting group and 39 men in the radicalprostatectomy group). The $\mathrm{P}$ value for the interaction between age and treatment with respect to death from prostate cancer was 0.10 .

Among men in the low-risk group, there was a significant absolute reduction in two of the three investigated end points: a reduction of 15.6 percentage points in the rate of death from any cause and 10.6 percentage points in the risk of metastases; the reduction of 3.8 percentage points in the rate of death from prostate cancer was not significant. Among men in the intermediate-risk group, there was a significant absolute reduction in all three end points: a reduction of 15.5 percentage points in overall mortality, 24.2 percentage points in the rate of death from prostate cancer, and 19.9 percentage points in the risk of metastases. Among men in the high-risk group, there was no significant reduction in absolute risk with respect to any of the end points. The $\mathrm{P}$ value for the interaction between tumor risk and treatment with respect to death from prostate cancer was 0.07 (Fig. 1 and 2 and Tables 1 and 2).

\section{DISCUSSION}

A significant absolute reduction in the rate of death from any cause, the rate of death from prostate cancer, and the risk of metastases in the radical-prostatectomy group continued after up to 23.2 years of follow-up (median, 13.4 years), with no evidence that these benefits diminished over time. In analyses according to age and tumor risk, the effects were more pronounced in men younger than 65 years of age and in men with intermediate-risk tumors. However, among men older than 65 years of age who underwent radical prostatectomy, there was a significantly decreased risk of metastases and need for palliative treatment. We 
observed a substantial difference in the prevalence of disease burden between the study groups.

In SPCG-4, the reduction in the rate of death attributed to radical prostatectomy increased with the number of years of follow-up, as shown by a relative reduction at 18 years of $44 \%$ and an increasing difference in rates of death from prostate cancer; this indicates that radical prostatectomy prevented deaths from tumors over a wide range of growth rates. Every third man who was assigned to watchful waiting had died from prostate cancer or from other causes, but with metastases present, whereas in the radical-prostatectomy group, every fifth man died. These results underscore the fact that our trial included men with tumors diagnosed before the advent of PSA screening. From 10 to 18 years of follow-up, the number needed to treat to prevent one death decreased from 20 to 8 in the whole cohort, and from 8 to 4 among men younger than 65 years of age.

At 18 years, the estimates of the risk of metastases and of death from other causes but with disease progression indicate that older men with a long life expectancy benefit from radical prostatectomy. Older men may have more urinary leakage after surgery, but erectile dysfunction may not profoundly affect quality of life in older men, since many of them are less sexually active and have a greater burden of erectile dysfunction preoperatively. ${ }^{11-15}$

Our analyses according to tumor risk aimed to provide evidence for the current debate about active surveillance. However, these analyses are merely hypothesis-generating: the numbers in each subgroup are low, and the risk categorization is less sophisticated than current standards. Given these caveats, the small absolute risk reduction in the rate of death from prostate cancer in the low-risk group at 18 years does not contradict current guidelines recommending active surveillance in men with low-risk prostate cancer. ${ }^{16}$ The modest reduction in absolute risk in the high-risk group differs from the results of the PIVOT study, which suggested a possible survival benefit only in men with intermediate-risk or high-risk tumors. ${ }^{2}$ However, there was evidence that a substantial proportion of men in the high-risk group in our trial had micrometastases at diagnosis (16 had lymph node-positive metastases), and therefore they did not undergo surgery. Thus, the difference between SPCG-4 and PIVOT may foremost reflect the different biologic domains of disease involved and possible differences in approaches to when to abstain from radical prostatectomy.

We used the prevalence of metastases and the use of palliative treatment as information about the burden of disease. As shown by previous studies ${ }^{12}$ and by the similar risk estimates associated with treatments for progressive disease and metastases, the use of palliative treatments in SPCG-4 closely followed the pattern of recurrent disease. A competing risk of cardiovascular deaths after androgen-deprivation therapy should therefore mainly be a problem among men with progressive disease. The finding that older men had an increased risk of death from causes other than prostate cancer may partly reflect this phenomenon. Metastases and the associated use of androgen-deprivation therapy are strongly associated with reduced quality of life. ${ }^{12,17}$ The life experience after the diagnosis of prostate cancer differs substantially between the two study groups and evolves over decades. Although the disease burden was large in the watchful-waiting group, a substantial 
proportion of men in this group did not have disease progression and did not receive palliative treatment. Therefore, there is a need to identify markers of aggressive disease.

One man died after surgery in the radical-prostatectomy group. Our follow-up to assess quality of life at a mean of 12.4 years showed a prevalence of erectile dysfunction of $84 \%$ in the radical-prostatectomy group and $80 \%$ in the watchful-waiting group; urinary leakage was reported in $41 \%$ and $11 \%$, respectively. Distress from these symptoms was reported as being significantly greater in men assigned to radical prostatectomy than watchful waiting. ${ }^{14}$

The strengths of our study include the randomized design, the completeness of follow-up, and the independent and blinded evaluation of the cause of death. Adherence to the assigned regimen was high. Our interpretation relies on estimates that are consistent over an extended follow-up. The subgroup analyses were not pre-specified in the protocol and are exploratory and sensitive to chance findings and limited power.

Extended follow-up 23 years after the initiation of the study corroborated a substantial reduction in the rate of death after radical prostatectomy. The number needed to treat to prevent one death has continued to decrease. The hypothesis-generating subgroup analyses and the large proportion of long-term survivors in the watchful-waiting group who never required palliative treatment provide support for active surveillance as an alternative in adequately selected groups. However, the overall long-term disease burden is also a reminder that factors other than survival should be considered when counseling men with localized prostate cancer; the risk of metastases and ensuing palliative treatments also affect quality of life.

\section{Supplementary Material}

Refer to Web version on PubMed Central for supplementary material.

\section{Acknowledgments}

Supported by grants from the Swedish Cancer Society (07 05 12, to Dr. Johansson; and 0905 12, to Dr. BillAxelson), the National Institutes of Health (1ROI CA 108746-O1A1, to Dr. Steineck), the Karolinska Institutet (distinguished professor award Dnr:2368/10-221, to Dr. Adami), and the Prostate Cancer Foundation and Percy Falk Foundation (both to Dr. Bill-Axelson).

\section{References}

1. Bill-Axelson A, Holmberg L, Ruutu M, et al. Radical prostatectomy versus watchful waiting in early prostate cancer. N Engl J Med. 2011; 364:1708-17. [PubMed: 21542742]

2. Wilt TJ, Brawer MK, Jones KM, et al. Radical prostatectomy versus observation for localized prostate cancer. N Engl J Med. 2012; 367:203-13. Erratum, N Engl J Med 2012;367:582. [PubMed: 22808955]

3. Holmberg L, Bill-Axelson A, Helgesen F, et al. A randomized trial comparing radical prostatectomy with watchful waiting in early prostate cancer. N Engl J Med. 2002; 347:781-9. [PubMed: 12226148]

4. Bill-Axelson A, Holmberg L, Filén F, et al. Radical prostatectomy versus watchful waiting in localized prostate cancer: the Scandinavian Prostate Cancer Group-4 randomized trial. J Natl Cancer Inst. 2008; 100:1144-54. [PubMed: 18695132] 
5. Harmer, MH., editor. TNM classification of malignant tumours. 3. Geneva: International Union against Cancer; 1978.

6. Brendler CB, Cleeve LK, Anderson EE, Paulson DF. Staging pelvic lymphadenectomy for carcinoma of the prostate risk versus benefit. J Urol. 1980; 124:849-50. [PubMed: 7441837]

7. Walsh PC, Lepor H, Eggleston JC. Radical prostatectomy with preservation of sexual function: anatomical and pathological considerations. Prostate. 1983; 4:473-85. [PubMed: 6889192]

8. Gleason, DF. Histologic grading and clinical staging of prostatic carcinoma. In: Tannenbaum, M., editor. Urologic pathology: the prostate. Philadelphia: Lea \& Febiger; 1977. p. 171-98.

9. Gray R. A class of K-sample tests for comparing the cumulative incidence of a competing risk. Ann Stat. 1988; 16:1141-54.

10. Kalbfleisch, JD.; Prentice, RL., editors. The statistical analysis of failure time data. 2. New York: John Wiley; 2002.

11. Steineck G, Helgesen F, Adolfsson J, et al. Quality of life after radical prostatectomy or watchful waiting. N Engl J Med. 2002; 347:790-6. [PubMed: 12226149]

12. Johansson E, Bill-Axelson A, Holmberg L, Onelöv E, Johansson JE, Steineck G. Time, symptom burden, androgen deprivation, and self-assessed quality of life after radical prostatectomy or watchful waiting: the Randomized Scandinavian Prostate Cancer Group Study Number 4 (SPCG-4) clinical trial. Eur Urol. 2009; 55:422-30. [PubMed: 18783877]

13. Bill-Axelson A, Garmo H, Holmberg L, et al. Long-term distress after radical prostatectomy versus watchful waiting in prostate cancer: a longitudinal study from the Scandinavian Prostate Cancer Group-4 Randomized Clinical Trial. Eur Urol. 2013; 64:920-8. [PubMed: 23465517]

14. Johansson E, Steineck G, Holmberg L, et al. Long-term quality-of-life outcomes after radical prostatectomy or watchful waiting: the Scandinavian Prostate Cancer Group-4 randomised trial. Lancet Oncol. 2011; 12:891-9. [PubMed: 21821474]

15. Stanford JL, Feng Z, Hamilton AS, et al. Urinary and sexual function after radical prostatectomy for clinically localized prostate cancer: the Prostate Cancer Outcomes Study. JAMA. 2000; 283:354-60. [PubMed: 10647798]

16. Horwich A, Hugosson J, de Reijke T, Wiegel T, Fizazi K, Kataja V. Prostate cancer: ESMO Consensus Conference Guidelines 2012. Ann Oncol. 2013; 24:1141-62. [PubMed: 23303340]

17. Sanda MG, Dunn RL, Michalski J, et al. Quality of life and satisfaction with outcome among prostate-cancer survivors. N Engl J Med. 2008; 358:1250-61. [PubMed: 18354103] 


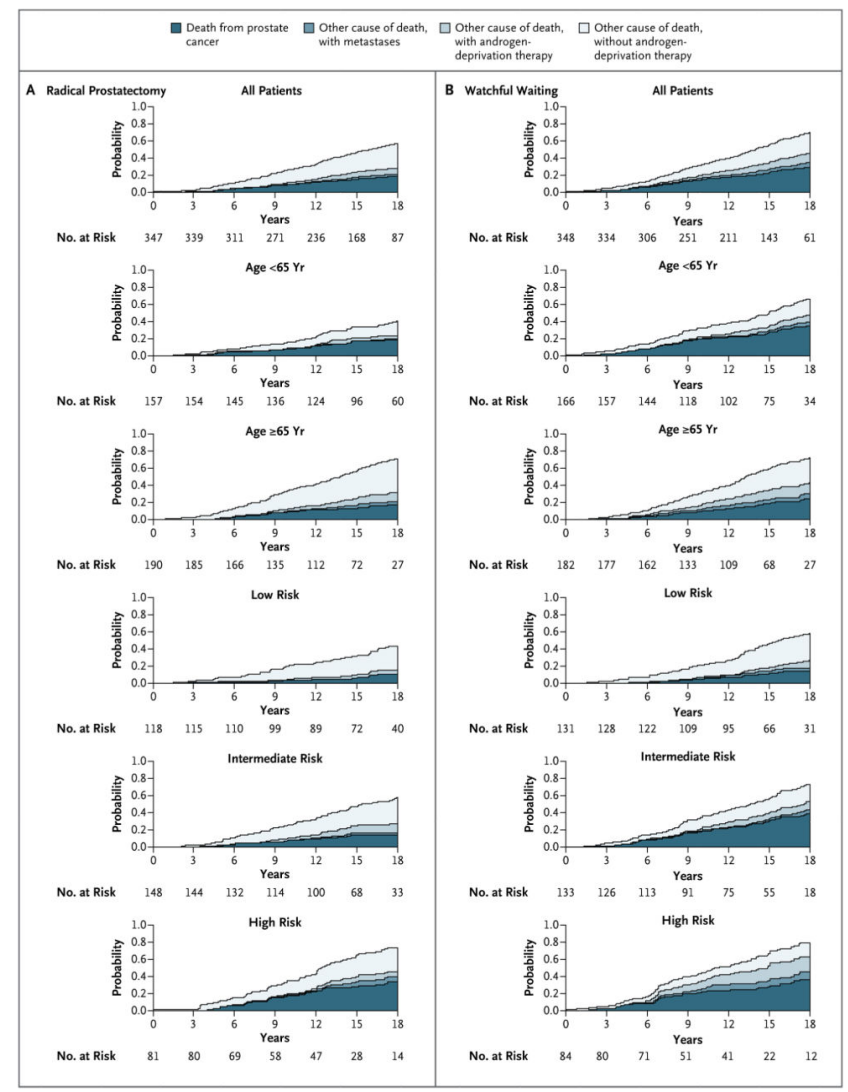

Figure 1.

Stacked Cumulative Incidence of Death from Any Cause, Death from Prostate Cancer, and the Development of Metastasis, According to Study Group, Age, and Risk Group. 


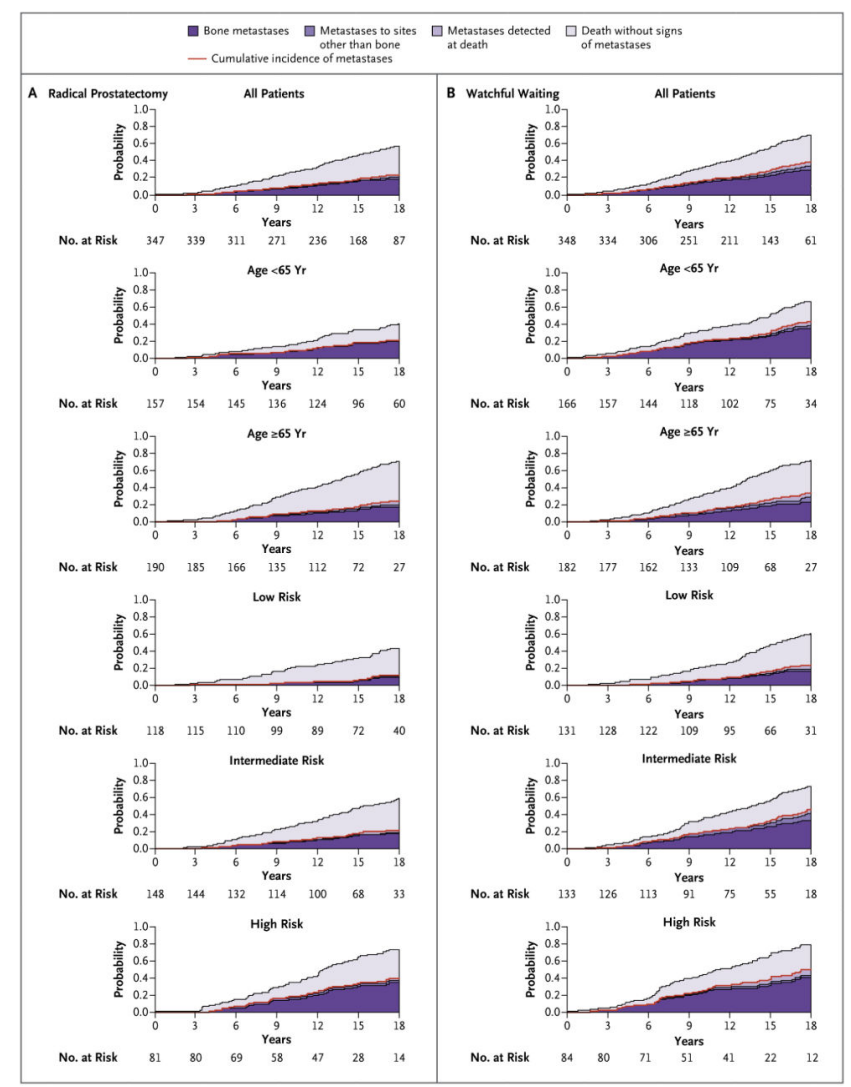

Figure 2.

Stacked Cumulative Incidence of Metastases and Death without Metastases, According to Study Group, Age, and Risk Group. 


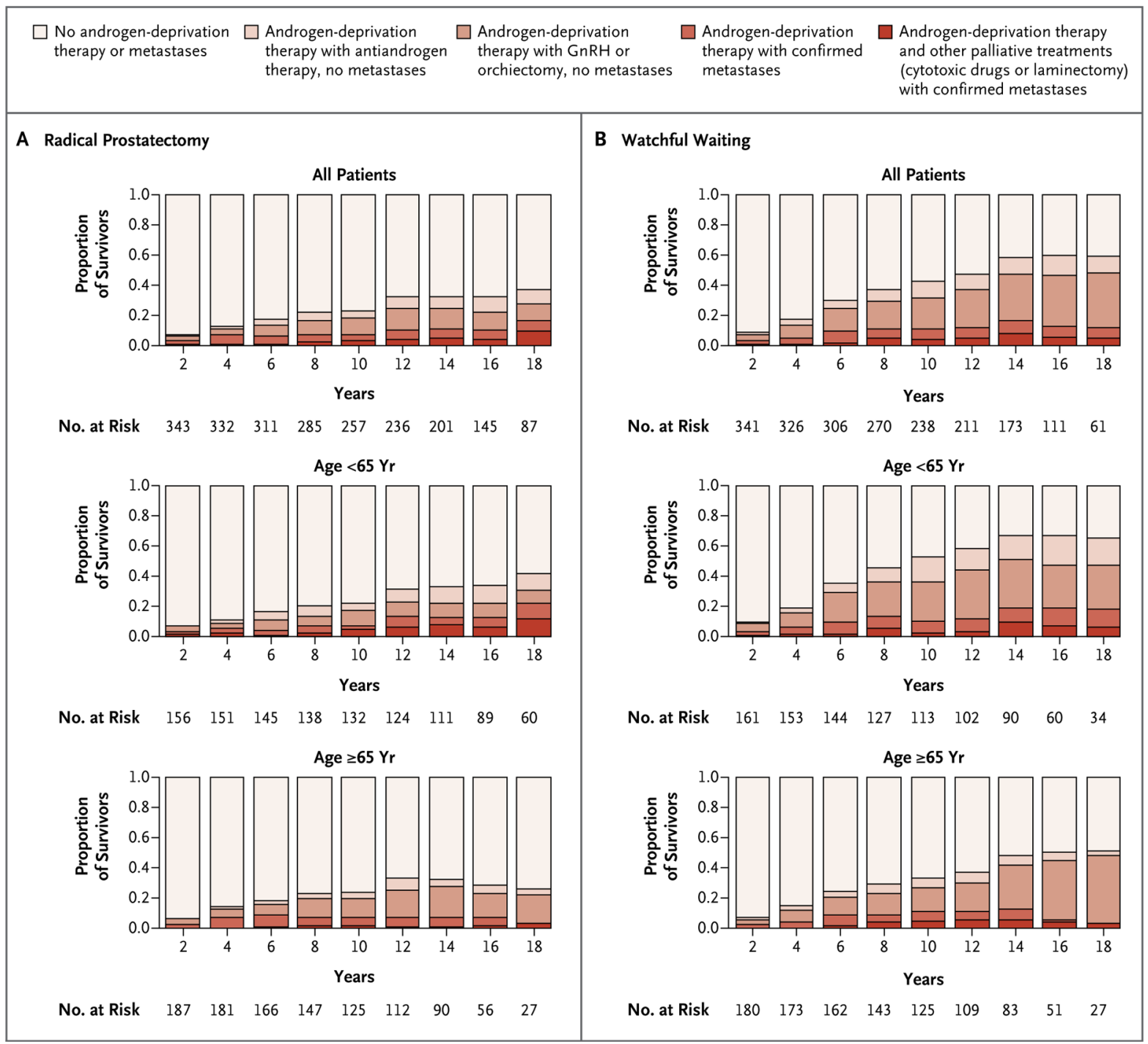

Figure 3. Prevalence of Metastases and Use of Palliative Treatment in Men Alive at Various Time Points since Randomization

The use of gonadotropin-releasing hormone (GnRH) analogues is considered to be medical castration, and orchiectomy is considered to be surgical castration. 


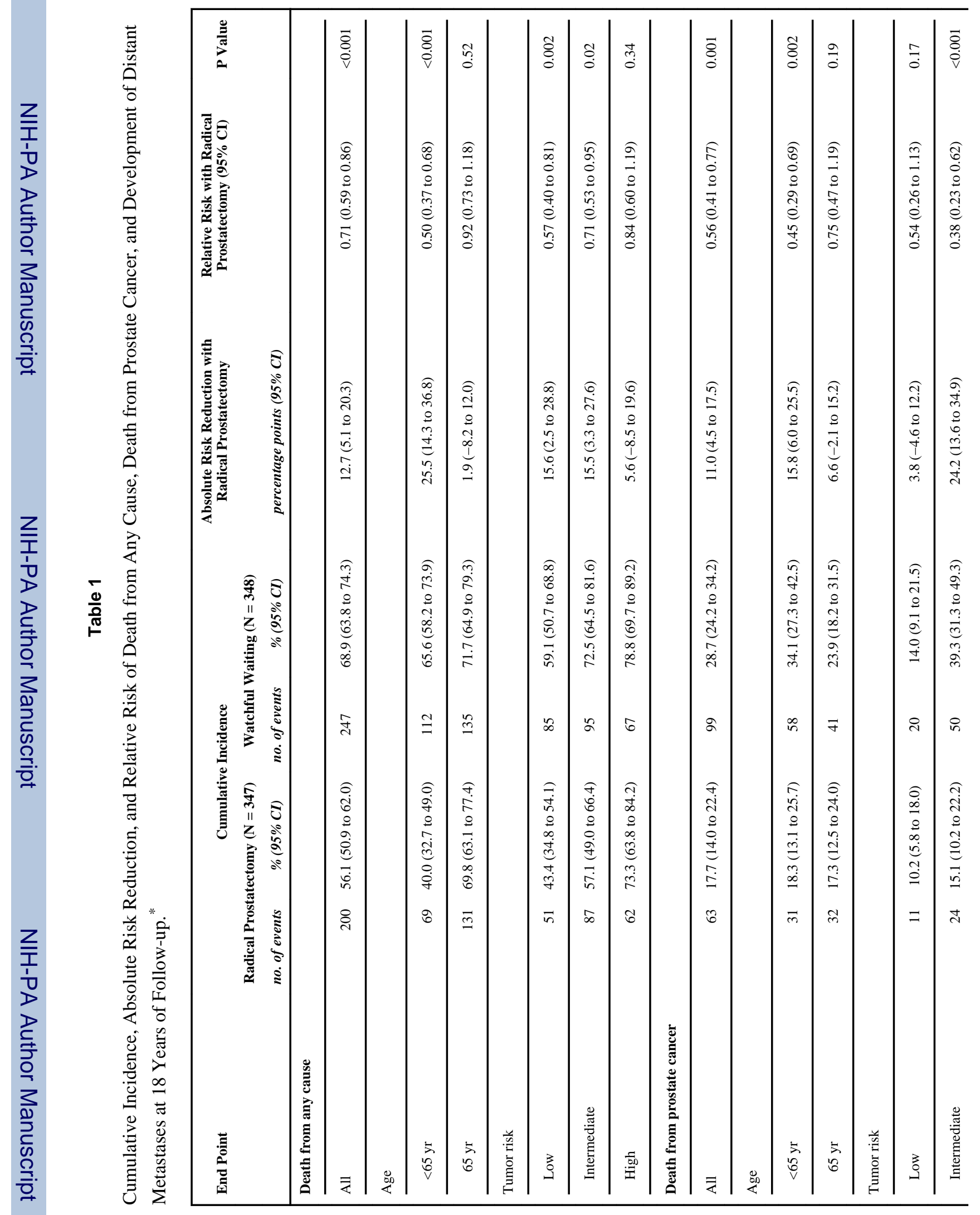




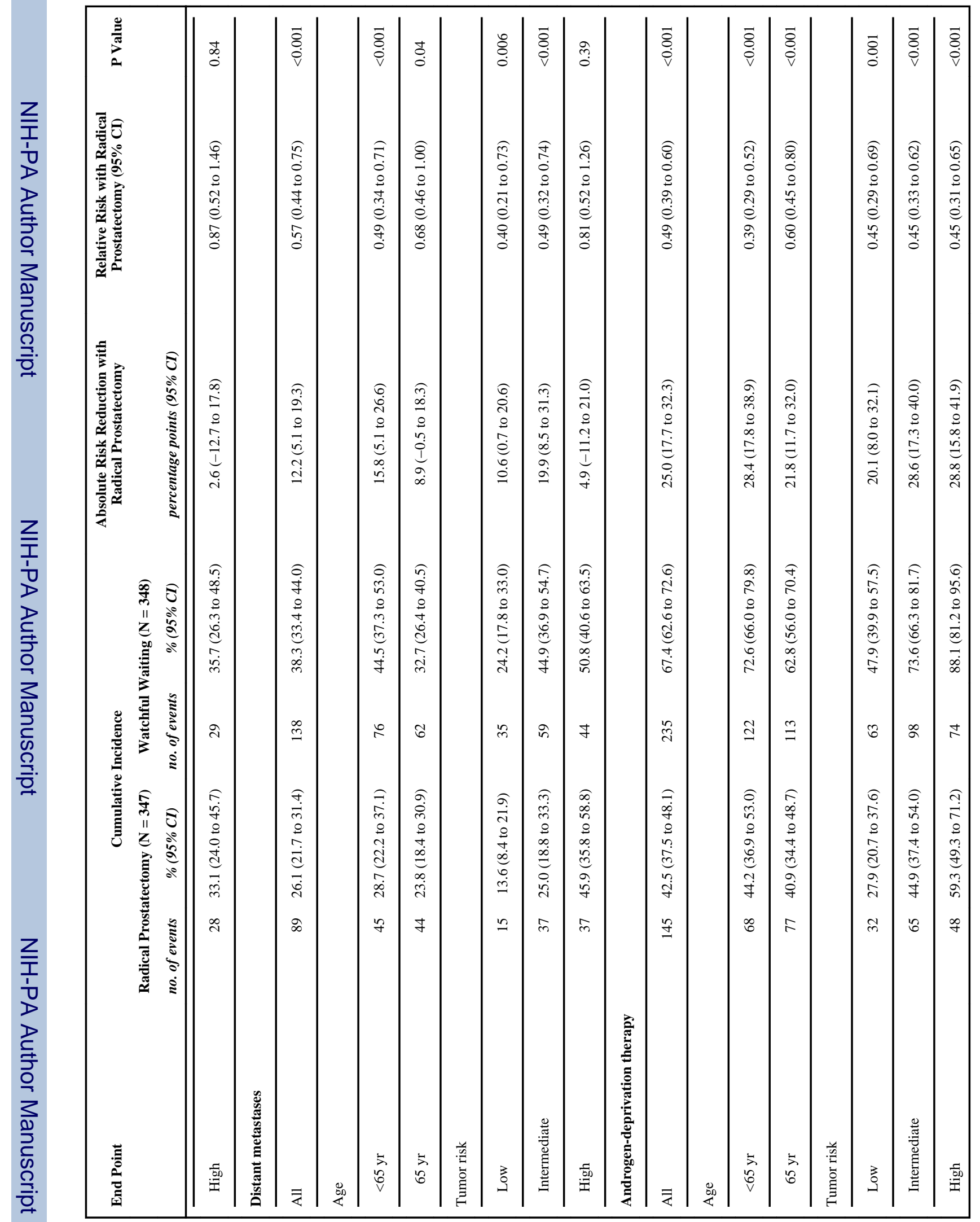



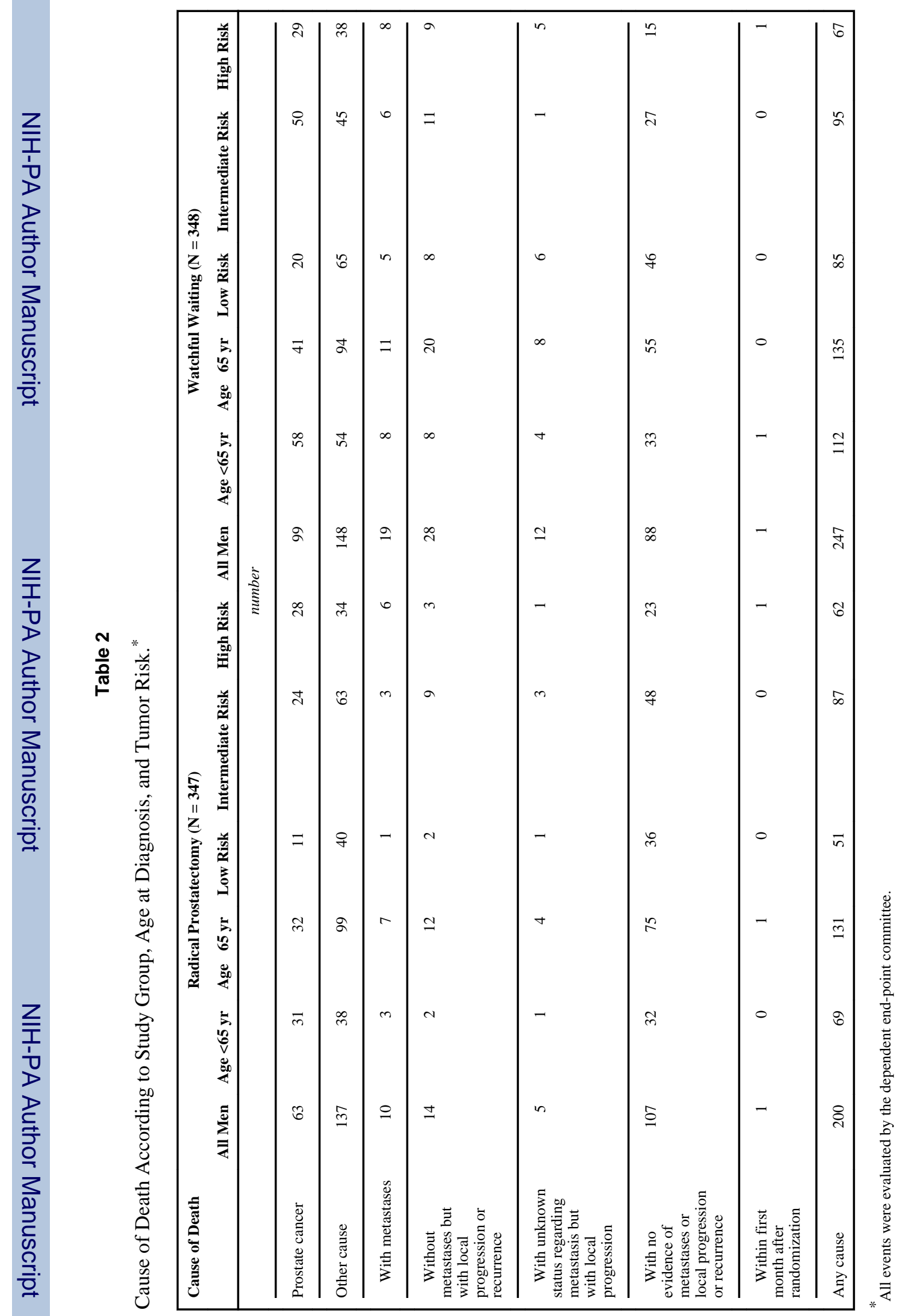

N Engl J Med. Author manuscript; available in PMC 2014 September 06. 